\title{
MAXIMAL DIFFERENTIAL UNIFORMITY POLYNOMIALS
}

\author{
YVES AUBRY, FABIEN HERBAUT, AND JOSÉ FELIPE VOLOCH
}

ABstract. We provide explicit infinite families of integers $m$ such that all the polynomials of $\mathbb{F}_{2^{n}}[x]$ of degree $m$ have maximal differential uniformity for $n$ large enough. We also prove a conjecture of the third author for these families.

\section{INTRODUCTION}

Throughout this paper $n$ is a positive integer and $q=2^{n}$. For a polynomial $f \in \mathbb{F}_{q}[x]$ we define the differential uniformity $\delta(f)$ following Nyberg $([6])$ :

$$
\delta(f):=\max _{(\alpha, \beta) \in \mathbb{F}_{q}^{*} \times \mathbb{F}_{q}} \sharp\left\{x \in \mathbb{F}_{q} \mid f(x+\alpha)+f(x)=\beta\right\} .
$$

When $\delta(f)=2$ the associated functions $f: \mathbb{F}_{q} \rightarrow \mathbb{F}_{q}$ are called APN (Almost Perfectly Nonlinear). These functions have been extensively studied as they offer good resistance against differential attacks (see [2]). Among them, those which are APN over infinitely many extensions of $\mathbb{F}_{q}$ have attracted special attention.

In the opposite direction the third author proved in [10] that most polynomials $f \in \mathbb{F}_{q}[x]$ of degree $m \equiv 0$ or $3(\bmod 4)$ have differential uniformity equal to $m-1$ or $m-2$, the largest possible for polynomials of degree $m$. Precisely, he proved that for a given integer $m>4$ such that $m \equiv 0(\bmod 4)$ $($ respectively $m \equiv 3(\bmod 4))$, if $\delta_{0}=m-2\left(\right.$ respectively $\left.\delta_{0}=m-1\right)$ then

$$
\lim _{n \rightarrow \infty} \frac{\sharp\left\{f \in \mathbb{F}_{2^{n}}[x] \mid \operatorname{deg}(f)=m, \delta(f)=\delta_{0}\right\}}{\sharp\left\{f \in \mathbb{F}_{2^{n}}[x] \mid \operatorname{deg}(f)=m\right\}}=1 .
$$

The first two authors extended this result to the second order differential uniformity in [1].

The following conjecture is also stated in [10]:

Conjecture 1.1. For a given integer $m>4$, there exists $\varepsilon_{m}>0$ such that for all sufficiently large $n$, if $f$ is a polynomial of degree $m$ over $\mathbb{F}_{2^{n}}$, for at least $\varepsilon_{m} 2^{2 n}$ values of $(\alpha, \beta) \in \mathbb{F}_{2^{n}}^{*} \times \mathbb{F}_{2^{n}}$ we have $\sharp\left\{x \in \mathbb{F}_{q} \mid f(x+\alpha)+f(x)=\right.$ $\beta\}=\delta(f)$.

Moreover, it was proved in [10] that all polynomials $f$ of degree 7 have maximal differential uniformity (that is here $\delta(f)=6$ ) if $n$ is large enough.

Date: October 12, 2018. 
The aim of this paper is to exhibit an infinite set $\mathcal{M}$ (defined below) of integers $m$ such that every polynomial $f \in \mathbb{F}_{2^{n}}[x]$ of degree $m$ has maximal differential uniformity if $n$ is large enough, that is $\delta(f)$ is equal to the degree of $D_{\alpha} f(x)=f(x+\alpha)+f(x)$, the derivative of $f$ with respect to $\alpha$. We stress that, for $m \in \mathcal{M}$, our results are much stronger than those of [10] as we prove maximality of differential uniformity for all polynomials of degree $m$, as opposed to most of them.

Definition. (Definition 3.10 and Proposition 3.11) We denote by $\mathcal{M}$ the set of the odd integers $m$ such that the unique polynomial $g$ satisfying $g(x(x+1))=$ $D_{1}\left(x^{m}\right)$ has distinct critical values.

We have that $m$ belongs to $\mathcal{M}$ if and only if for any $\zeta_{1}$ and $\zeta_{2}$ in $\overline{\mathbb{F}}_{2} \backslash\{1\}$, the equalities $\zeta_{1}^{m-1}=\zeta_{2}^{m-1}=\left(\frac{1+\zeta_{1}}{1+\zeta_{2}}\right)^{m-1}=1$ imply $\zeta_{1}=\zeta_{2}$ or $\zeta_{1}=\zeta_{2}^{-1}$.

Now we can state our main results.

Theorem. (Theorem 5.3 and Theorem 5.7) Let $m \in \mathcal{M}$ such that $m \equiv 7$ (mod 8). Then for $n$ sufficiently large, for all polynomials $f \in \mathbb{F}_{2^{n}}[x]$ of degree $m$ we have $\delta(f)=m-1$. Furthermore, Conjecture 1.1. is true for such integers $m$.

For example, we will prove that the previous theorem applies for the integers $m \in\{7,23,39,47,55,79,87,95,111,119,135,143,159,167,175,191,199\}$ (see Example 3.16). We also provide explicit infinite families of such integers $m$, namely the integers $m=2 \ell^{2 k+1}+1$ for $k \geqslant 0$ and $\ell \in\{3,11,19,23,43,47$, $59,67,71,79,83,103,107,131,139,151,163,167,179,191,199\}$ (see Corollary 5.4).

When $m$ is congruent to 3 modulo 8 , we also obtain some results but we have conditions on the parity of $n$ or we have to remove some polynomials.

Theorem. (Theorem 5.5)

Let $m \in \mathcal{M}$ such that $m \geqslant 7$ and $m \equiv 3(\bmod 8)$.

(i) For $n$ even and sufficiently large and for all polynomials $f \in \mathbb{F}_{2^{n}}[x]$ of degree $m$ we have $\delta(f)=m-1$.

(ii) For $n$ sufficiently large and for all polynomials $f=\sum_{i=0}^{m} a_{m-i} x^{i}$ in $\mathbb{F}_{2^{n}}[x]$ of degree $m$ such that $a_{1}^{2}+a_{0} a_{2} \neq 0$, we have $\delta(f)=m-1$.

We also provide infinite families of integers $m \equiv 3(\bmod 8)$ for which the previous theorem applies, namely the integers $m=2 \ell^{k}+1$ for $k \geqslant 1$ and $\ell \in\{17,41,97,113,137,193\}$ and the integers $m=2 \ell^{2 k}+1$ for $k \geqslant 1$ and $\ell \in\{23,47,71,79,103,151,167,191,199\}$ (see Corollary 5.6).

Let us explain the strategy of the proofs of the above theorems which has important similarities to that of [10] and [1]. For simplicity we consider in this sketch the case where $m$ is congruent to 7 modulo 8 .

If $f \in \mathbb{F}_{q}[x]$ is a polynomial of degree $m$ and if $\alpha \in \mathbb{F}_{q}^{*}$, we introduce the unique polynomial $L_{\alpha} f$ of degree $d=(m-1) / 2$ such that $L_{\alpha} f(x(x+\alpha))=$ 
$D_{\alpha} f(x)$ (see Proposition 2.3) . We consider the splitting field $F$ of the polynomial $L_{\alpha} f(x)-t$ over the field $\mathbb{F}_{q}(t)$ with $t$ transcendental over $\mathbb{F}_{q}$ and set $\mathbb{F}_{q}^{F}$ be the algebraic closure of $\mathbb{F}_{q}$ in $F$. The Galois groups $G=\operatorname{Gal}\left(F / \mathbb{F}_{q}(t)\right)$ and $\bar{G}=\operatorname{Gal}\left(F / \mathbb{F}_{q}^{F}(t)\right)$ are respectively the arithmetic and geometric monodromy groups of $L_{\alpha} f$.

If $u_{0}, \ldots, u_{d-1}$ are the roots of $L_{\alpha} f(x)=t$, then we will denote by $x_{i}$ a root of $x^{2}+\alpha x=u_{i}$. So the $2 d$ elements $x_{0}, x_{0}+\alpha, \ldots, x_{d-1}, x_{d-1}+\alpha$ are the solutions of $D_{\alpha} f(x)=t$. Thus we consider $\Omega=\mathbb{F}_{q}\left(x_{0}, \ldots, x_{d-1}\right)$ the compositum of the fields $F\left(x_{i}\right)$ and $\mathbb{F}_{q}^{\Omega}$ the algebraic closure of $\mathbb{F}_{q}$ in $\Omega$. We set also $\Gamma=\operatorname{Gal}(\Omega / F)$ and $\bar{\Gamma}=\operatorname{Gal}\left(\Omega / F \mathbb{F}_{q}^{\Omega}\right)$. Then we have the following diagram:

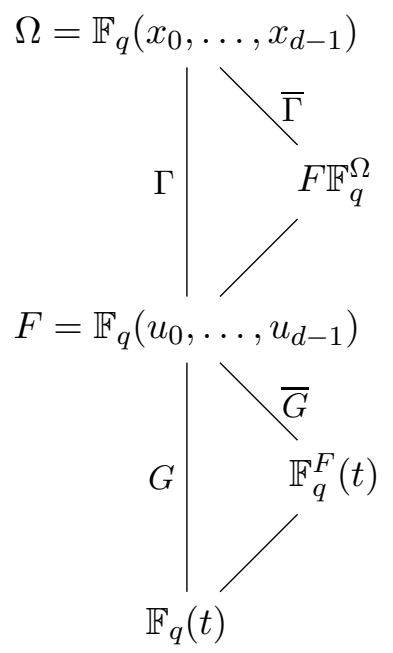

When the integer $m$ belongs to $\mathcal{M}$ and is congruent to 7 modulo 8 we prove that for $n$ sufficiently large and for any polynomial $f \in \mathbb{F}_{2^{n}}[x]$ of degree $m$, there exists $\alpha$ in $\mathbb{F}_{2^{n}}^{*}$ such that:

(1) $L_{\alpha} f$ is Morse

(2) the equation $x^{2}+\alpha x=\frac{b_{1}}{b_{0}}$ has a solution in $\mathbb{F}_{2^{n}}$.

Now, condition (1) implies by Proposition 4.1 that the extension $F / \mathbb{F}_{q}(t)$ is regular. Condition (1) and (2) imply by Proposition 4.6 that the extension $\Omega / F$ is regular. It enables us to apply Chebotarev density theorem (see Proposition 5.1) to obtain, for $n$ sufficiently large depending only on $m$, the existence of $\beta \in \mathbb{F}_{2^{n}}$ such that the polynomial $D_{\alpha} f(x)+\beta$ splits in $\mathbb{F}_{2^{n}}[x]$ with no repeated factors. The differential uniformity of $f$ is thus equal to the degree of $D_{\alpha} f$.

The paper is organized as follows. Section 2 is devoted to the study of the operator $L_{\alpha}$. Section 3 provides a detailed exposition of Morse polynomials in even characteristic. According to the appendix by Geyer in [5], Morse polynomials in this context are polynomials of odd degree satisfying two 
conditions: their critical points are non degenerate and their critical values are distinct. The first condition leads to the study of the number of $\alpha$ such that the resultant of the derivative $\left(L_{\alpha} f\right)^{\prime}$ with the second Hasse-Schmidt derivative $\left(L_{\alpha} f\right)^{[2]}$ does not vanish (Proposition 3.2). We give upper bounds for the number of exceptions in terms of $m$.

By contrast, we need additional requirements on $m$ to guarantee that for enough $\alpha$ the polynomial $L_{\alpha} f$ has distinct critical values (see Proposition 3.6). Precisely, we will make the assumption that $L_{1}\left(x^{m}\right)$ has distinct critical values, this is that $m$ belongs to $\mathcal{M}$ (Definition 3.10). We complete Section 3 by exhibiting some families of infinitely many integers belonging to $\mathcal{M}$.

Section 4 is devoted to the study of the Galois groups $G, \bar{G}, \Gamma$ and $\bar{\Gamma}$. We prove in Proposition 4.6 that if the equation $x^{2}+\alpha x=\frac{b_{1}}{b_{0}}$ has a solution in $\mathbb{F}_{2^{n}}$ i.e. if $\operatorname{Tr}_{\mathbb{F}_{2^{n}} / \mathbb{F}_{2}}\left(\frac{b_{1}}{b_{0} \alpha^{2}}\right)=0$ then the extension $\Omega / F$ is regular. The different expressions of $b_{1} / b_{0}$ we have obtained in Lemma 2.5, depending on the congruence of $m$ modulo 8 , induce differences in the treatment.

Section [5] deals with the Chebotarev density theorem and contains the statements and the proofs of the main results.

Let us stress the main difference between the common approach of [10] and [1] and the approach of the present paper. For simplicity, we consider again that $m \equiv 7(\bmod 8)$. In [10] and [1], one of the key steps is to fix $\alpha_{1}, \ldots, \alpha_{k}$ in $\mathbb{F}_{2^{n}}$ and to obtain a lower bound depending on $n$ for the number of polynomials $f$ in $\mathbb{F}_{2^{n}}[x]$ such that at least one of the $L_{\alpha_{i}} f$ is Morse. By contrast, we prove here that for $n$ sufficiently large and for any polynomial $f$ of degree $m$ in $\mathbb{F}_{2^{n}}[x]$ there exists $\alpha$ such that $L_{\alpha} f$ is Morse.

\section{The associated Polynomial $L_{\alpha} f$}

Let $f \in \mathbb{F}_{q}[x]$ be a polynomial of degree $m \geqslant 7$ (the cases where $m<7$ are handled in [10]) and $\alpha \in \mathbb{F}_{q}^{*}$. The derivative of a polynomial $f \in \mathbb{F}_{q}[x]$ along $\alpha$ is defined by:

$$
D_{\alpha} f(x)=f(x)+f(x+\alpha) .
$$

If we set $f=\sum_{k=0}^{m} a_{m-k} x^{k}$, a straightforward computation gives that $D_{\alpha} f=\sum_{k=0}^{m} c_{m-k} x^{k}$ where $c_{k}=a_{k}+\sum_{i=m-k}^{m} a_{m-i}\left(\begin{array}{c}i \\ m-k\end{array}\right) \alpha^{i-m+k}$. As we work over an even characteristic field, we have $c_{0}=a_{0}+a_{0}=0, c_{1}=m \alpha a_{0}$ and $c_{2}=(m-1) \alpha a_{1}+\left(\begin{array}{c}m \\ 2\end{array}\right) \alpha^{2} a_{0}$. We deduce the following proposition.

Proposition 2.1. Let $f \in \mathbb{F}_{q}[x]$ be a polynomial of degree $m$. If $m$ is odd then the degree of $D_{\alpha} f$ is $m-1$. If $m$ is even then the degree of $D_{\alpha} f$ is less than or equal to $m-2$, and equal to $m-2$ if and only if $a_{1}+a_{0} \alpha\left(\begin{array}{c}m \\ 2\end{array}\right) \neq 0$. $d$.

In the whole paper, we will associate to any integer $m$ the following integer 
Definition 2.2. Let $m$ be an integer. Suppressing in our notation the dependence on $m$, we set $d=(m-1) / 2$ if $m$ is odd and $d=(m-2) / 2$ is $m$ is even.

\subsection{Existence of $L_{\alpha} f$.}

Proposition 2.3. Let $\alpha \in \mathbb{F}_{q}^{*}$ and let $f \in \mathbb{F}_{q}[x]$ be a polynomial of degree $m$. Then there exists a unique polynomial $g \in \mathbb{F}_{q}[x]$ of degree less than or equal to d such that

$$
D_{\alpha} f(x)=g(x(x+\alpha)) .
$$

Furthermore, the map $L_{\alpha}: f \longmapsto g$ is linear and its restriction to the subspace of polynomials of degree at most $m$ is surjective onto the subspace of polynomials of degree at most $d$.

Proof. The proof is similar to that of Proposition 2.2. of [1] dealing with the set $\Lambda_{k}$ of roots of multiplicity $k$ of $D_{\alpha} f$ and noticing that $x \mapsto x+\alpha$ is an involution of each set $\Lambda_{k}$. The surjectivity of $L_{\alpha}$ follows from the fact that the kernel of the restriction of $L_{\alpha}$ to the space of polynomials of degree at most $m$ is the subspace of polynomials $g(x(x+\alpha))$ where $g \in \mathbb{F}_{q}[x]$ has degree at most $[m / 2]$ (see Lemma 2.3. of [1]).

\subsection{The coefficients $b_{i}$ of $L_{\alpha} f$.}

Let $f=\sum_{i=0}^{m} a_{m-i} x^{i} \in \mathbb{F}_{q}[x]$ be a polynomial of degree $m$ and $L_{\alpha} f=$ $\sum_{i=0}^{d} b_{d-i} x^{i}$ be the associated polynomial of degree $d$ when $m$ is odd and of degree less than or equal to $d$ otherwise (see Proposition 2.1). To obtain information on the coefficients $b_{i}$, one can consider the triangular linear system with coefficients 1 on the diagonal arising when identifying the coefficients of $x^{2 d}, x^{2 d-2}, \ldots, x^{2}, x^{0}$ in $g(x(x+\alpha))$ and in $D_{\alpha} f$. Note that this approach proves again the unicity of $g$ claimed in Proposition 2.3.

More precisely, a necessary condition for the term $b_{s} x^{t}$ to appear in $g(x(x+\alpha))$ is that $d-t \leqslant s \leqslant d-t / 2$. In this case, it appears with the coefficient $\left(\begin{array}{c}d-s \\ t-d+s\end{array}\right) \alpha^{2(d-s)-t}$. So for each integer $k$ between 0 and $d$, identifying the coefficient of $x^{2(d-k)}$ in $g(x(x+\alpha))$ and in $D_{\alpha} f(x)$ gives

$$
\sum_{s=\operatorname{Max}\{0,2 k-d\}}^{k}\left(\begin{array}{c}
d-s \\
2 k-2 s
\end{array}\right) \alpha^{2 k-2 s} b_{s}=\sum_{i=2 d-2 k+1}^{m}\left(\begin{array}{c}
i \\
2 d-2 k
\end{array}\right) \alpha^{i-2 d+2 k} a_{m-i} .
$$

We consider the polynomial ring $\mathbb{F}_{2}\left[\alpha, a_{0}, \ldots, a_{m}\right]$ where $\alpha, a_{0}, \ldots, a_{m}$ are indeterminates with the degree $w$ such that $w(\alpha)=1$ and $w\left(a_{j}\right)=j$. It means that the monomial $\alpha^{d_{\alpha}} a_{0}^{d_{0}} a_{1}^{d_{1}} a_{2}^{d_{2}} \ldots a_{m}^{d_{m}}$ has degree $d_{\alpha}+d_{1}+2 d_{2}+$ $\cdots+m d_{m}$. Then using the triangular system obtained from (1) and an induction on $k$ prove the following homogeneity result. 
Lemma 2.4. For all integers $i$ such that $0 \leqslant i \leqslant d$ we have $b_{i} \in \mathbb{F}_{2}\left[\alpha, a_{0}, \ldots, a_{m}\right]$ which is an homogeneous polynomial of degree $2 i+1$ if $m$ is odd and of degree $2 i+2$ if $m$ is even, when considering the degree $w$ such that $w(\alpha)=1$ and $w\left(a_{j}\right)=j$.

The relations (11) also provide expressions of the first coefficients $b_{0}, b_{1}, \ldots$ of $L_{\alpha} f$ depending on the congruence class of $m$ modulo 8 , as made explicit in the next lemma which will be needed in the proof of Theorem 5.3. Note that formulas for $b_{1} / b_{0}$ appeared in [10] as well, but the last two had misprints.

Lemma 2.5. Let $m$ be an integer. If $m \equiv 0(\bmod 4)$ then $b_{0}=a_{1} \alpha$ and if $m \equiv 3(\bmod 4)$ then $b_{0}=a_{0} \alpha$. Moreover, we have the following expressions of $b_{1} / b_{0}$ depending on the congruence of $m$ :

\begin{tabular}{cc}
\hline$m(\bmod 8)$ & $b_{1} / b_{0}$ \\
3 & $\alpha^{2}+\frac{a_{1} \alpha+a_{2}}{a_{0}}$ \\
7 & $\frac{a_{1} \alpha+a_{2}}{a_{0}}$ \\
0 & $\frac{a_{2} \alpha+a_{3}}{a_{1}}$ \\
4 & $\alpha^{2}+\frac{a_{0} \alpha^{3}+a_{2} \alpha+a_{3}}{a_{1}}$ \\
\hline
\end{tabular}

\section{For almost every $\alpha$ the polynomial $L_{\alpha} f$ is Morse}

We will focus now on polynomials $f$ of degree $m \equiv 3(\bmod 4)$ and thus, for nonzero $\alpha$, on polynomials $L_{\alpha} f$ of odd degree $d=(m-1) / 2$.

3.1. Morse polynomials in even characteristic. We consider the following notion of Morse polynomial given in all characteristic by Geyer in an appendix to the paper [5].

Definition 3.1. Let $K$ be a field of characteristic $p \geqslant 0$. We say that a polynomial $g$ over $K$ is Morse if the three following conditions hold:

(a) the critical points of $g$, i.e the zeroes of $g^{\prime}$, are non degenerate,

(b) the critical values of $g$ are distinct, i.e. $g^{\prime}(\tau)=g^{\prime}(\eta)=0$ and $g(\tau)=g(\eta)$ imply $\tau=\eta$,

(c) if $p>0$, then the degree of $g$ is not divisible by $p$.

These conditions are chosen such that $g$ corresponds to a covering with maximum Galois group, that is $\mathrm{Gal}(g(t)-x, K(x))$ is the symmetric group $\mathfrak{S}_{d}$ where $d$ is the degree of $g$ (see Proposition 4.2 in [5]). In the case where $p>0$, the loci of non-Morse polynomials is described in the same appendix.

Let us sum up the situation in the case where $p=2$. In this case one has to introduce the Hasse-Schmidt derivative $g^{[2]}$ which is defined by the equality $g(t+u) \equiv g(t)+g^{\prime}(t) u+g^{[2]}(t) u^{2}\left(\bmod u^{3}\right)$ where $u$ and $t$ are independent variables. If $g=\sum_{i=0}^{d} b_{d-i} x^{i}$ is a degree $d$ polynomial of $\mathbb{F}_{q}[x]$ 
with $q$ a power of 2 , then the condition (a) above is fulfilled if and only if $g^{\prime}$ and $g^{[2]}$ have no common roots, that is if and only if the resultant

$$
R:=\operatorname{Res}\left(g^{\prime}, g^{[2]}\right) \in \mathbb{F}_{2}\left[b_{0}, \ldots, b_{d}\right]
$$

does not vanish. And the condition (b) above is fulfilled if and only if

$$
\Pi(g):=\prod_{i \neq j}\left(g\left(\tau_{i}\right)-g\left(\tau_{j}\right)\right)
$$

does not vanish, where $\tau_{1}, \ldots, \tau_{\left[\frac{d-1}{2}\right]}$ are the (double) roots of $g^{\prime}$. Using the theorem on symmetric functions, one can obtain an expression of $\Pi(g)$ depending on the coefficients $b_{0}, \ldots, b_{d}$ of $g$.

In order to calculate the second order Hasse-Schmidt derivative, we will make use of the following Lucas theorem about binomial coefficients (see for instance the introduction of [4]). For $p$ a prime number, write $m=$ $m_{0}+m_{1} p+m_{2} p^{2}+\cdots+m_{r} p^{r}$ and $k=k_{0}+k_{1} p+k_{2} p^{2}+\cdots+k_{r} p^{r}$ in base $p$. Then we have $\left(\begin{array}{c}m \\ k\end{array}\right) \equiv\left(\begin{array}{c}m_{0} \\ k_{0}\end{array}\right)\left(\begin{array}{c}m_{1} \\ k_{1}\end{array}\right) \cdots\left(\begin{array}{c}m_{r} \\ k_{r}\end{array}\right)(\bmod p)$.

3.2. The condition (a). In order to bound the number of $\alpha$ such that the critical values of $L_{\alpha} f$ are non degenerate, we study in this subsection $\operatorname{Res}\left(\left(L_{\alpha} f\right)^{\prime},\left(L_{\alpha} f\right)^{[2]}\right) \in \mathbb{F}_{2}\left[a_{0}, \ldots, a_{m}\right]$.

We will need three lemmas to succeed in doing so. Lemma 3.3 enables us to study $\tilde{R}:=\operatorname{Res}\left(\left(D_{\alpha} f\right)^{\prime},\left(D_{\alpha} f\right)^{[2]}\right)$ rather than $\operatorname{Res}\left(\left(L_{\alpha} f\right)^{\prime},\left(L_{\alpha} f\right)^{[2]}\right)$. Then Lemma 3.4 gives a result about the homogeneity and the degree of this polynomial if it is nonzero. To prove its non nullity we evaluate it in $a_{0}=1, a_{1}=\cdots=a_{m}=0$ which amounts to determining in Lemma 3.5 if the polynomial $x^{m}$ has non degenerate critical points.

Proposition 3.2. Let $m \geqslant 7$ such that $m \equiv 3(\bmod 4)$ and let $f(x)=$ $\sum_{k=0}^{m} a_{m-k} x^{k}$ be a polynomial of $\mathbb{F}_{q}[x]$ of degree $m$. Then the critical points of $L_{\alpha} f$ are non degenerate except for at most $m(m-3)$ values of $\alpha \in \overline{\mathbb{F}}_{2}$.

Lemma 3.3. Let $f \in \mathbb{F}_{q}[x]$ be a polynomial. For all $\alpha \in \mathbb{F}_{q}^{*}$ the polynomials $\left(L_{\alpha} f\right)^{\prime}$ and $\left(L_{\alpha} f\right)^{[2]}$ have a common root in $\overline{\mathbb{F}}_{2}$ if and only if the polynomials $\left(D_{\alpha} f\right)^{\prime}$ and $\left(D_{\alpha} f\right)^{[2]}$ have a common root in $\overline{\mathbb{F}}_{2}$.

Proof. Since $D_{\alpha} f=L_{\alpha} f \circ T_{\alpha}$ where $T_{\alpha}(x):=x(x+\alpha)$, we can prove the two following equalities:

$$
\left(D_{\alpha} f\right)^{\prime}=\alpha\left(L_{\alpha} f\right)^{\prime} \circ T_{\alpha}
$$

and

$$
\left(D_{\alpha} f\right)^{[2]}=\left(L_{\alpha} f \circ T_{\alpha}\right)^{[2]}=\left(L_{\alpha} f\right)^{\prime} \circ T_{\alpha}+\alpha^{2}\left(L_{\alpha} f\right)^{[2]} \circ T_{\alpha} .
$$

The result follows.

Lemma 3.4. Let $m \geqslant 7$ such that $m \equiv 3(\bmod 4)$ and let $f=\sum_{k=0}^{m} a_{m-k} x^{k}$ in $\mathbb{F}_{2}\left[a_{0}, \ldots, a_{m}\right][x]$. Consider the degree $w$ defined by $w(\alpha)=1$ and $w\left(a_{i}\right)=$ $i$ for any $i$ and consider also the degree $\tilde{w}$ defined by $\tilde{w}(\alpha)=0$ and $\tilde{w}\left(a_{i}\right)=1$. 
Then the resultant $\operatorname{Res}\left(\left(D_{\alpha} f\right)^{\prime},\left(D_{\alpha} f\right)^{[2]}\right)$ in the variable $x$, if it is nonzero, is an homogeneous polynomial of $\mathbb{F}_{2}\left[a_{0}, \ldots, a_{m}, \alpha\right]$ of degree $m(m-3)$ when considering the degree $w$ and is an homogeneous polynomial of degree $2(m-$ 3) when considering the degree $\tilde{w}$.

Proof. As $f(x)=\sum_{k=0}^{m} a_{m-k} x^{k}$ and $f(x+\alpha)=\sum_{k=0}^{m} a_{m-k}(x+\alpha)^{k}$, these two polynomials are homogeneous of degree $m$ for the degree $w$ such that $w(\alpha)=1, w\left(a_{i}\right)=i$ and $w(x)=1$. It follows that $\left(D_{\alpha} f\right)^{\prime}$ and $\left(D_{\alpha} f\right)^{[2]}$ are homogeneous of degree respectively $m-1$ and $m-2$ for the degree $w$. Using the formulae of $D_{\alpha} f$ given in Section 2, we have:

$$
D_{\alpha} f(x)=\alpha a_{0} x^{m-1}+a_{0} \alpha^{2} x^{m-2}+\left(a_{0} \alpha^{3}+a_{1} \alpha^{2}+a_{2} \alpha\right) x^{m-3}+\cdots
$$

The polynomial $\left(D_{\alpha} f\right)^{\prime}$ has degree $m-3$ in the variable $x$ since $m$ is odd and its leading coefficient is $a_{0} \alpha^{2}$. The polynomial $\left(D_{\alpha} f\right)^{[2]}$ has also degree $m-3$ in the variable $x$ since it can be shown that $\left(x^{k}\right)^{[2]}=\left(\begin{array}{l}k \\ 2\end{array}\right) x^{k-2}$ using the binomial theorem, the above Lucas theorem and the congruence of $m$. Its leading coefficient is $a_{0} \alpha$.

Thus we can set $\left(D_{\alpha} f\right)^{\prime}=\sum_{i=0}^{m-3} d_{i} x^{m-3-i}$ and $\left(D_{\alpha} f\right)^{[2]}=\sum_{i=0}^{m-3} e_{i} x^{m-3-i}$ where $d_{i}, e_{i} \in \mathbb{F}_{2}\left[a_{0}, \ldots, a_{m}, \alpha\right]$ are such that $w\left(d_{i}\right)=i+2$ and $w\left(e_{i}\right)=i+1$. Thus the resultant Res $\left(\left(D_{\alpha} f\right)^{\prime},\left(D_{\alpha} f\right)^{[2]}\right)$ in the variable $x$, if it is nonzero, is an homogeneous polynomial of $\mathbb{F}_{2}\left[a_{0}, \ldots, a_{m}, \alpha\right]$ of degree $m(m-3)$ for the degree $w$. For the second homogeneity result claimed, note that this resultant is a sum of $2(m-3)$ products of the coefficients $d_{i}$ and $e_{i}$, and each one of them is a linear combination in the $a_{0}, \ldots, a_{m}$.

Lemma 3.5. Let $m \geqslant 7$ such that $m \equiv 3(\bmod 4)$ and let $f=x^{m}$. For all $\alpha \in \mathbb{F}_{q}^{*}$ the critical points of $L_{\alpha} f$ are non degenerate.

Proof. Using Lemma 3.3 we look for the common roots of $\left(D_{\alpha} f\right)^{\prime}$ and $\left(D_{\alpha} f\right)^{[2]}$. We compute $\left(D_{\alpha} f\right)^{\prime}=(x+\alpha)^{m-1}+x^{m-1}$ and $\left(D_{\alpha} f\right)^{[2]}=$ $(x+\alpha)^{m-2}+x^{m-2}$. Hence, if $\omega \in \overline{\mathbb{F}}_{2}$ was a common root of $\left(D_{\alpha} f\right)^{\prime}$ and $\left(D_{\alpha} f\right)^{[2]}$ then we would have $((\omega+\alpha) / \omega)^{m-1}=((\omega+\alpha) / \omega)^{m-2}=1$, and so $\alpha=0$.

Now we are enable to prove Proposition 3.2 .

Proof. Lemma 3.3 enables us to study $\tilde{R}:=\operatorname{Res}\left(\left(D_{\alpha} f\right)^{\prime},\left(D_{\alpha} f\right)^{[2]}\right)$ rather than Res $\left(\left(L_{\alpha} f\right)^{\prime},\left(L_{\alpha} f\right)^{[2]}\right)$. Using the homogeneity results given by Lemma 3.4 we know that there is at most one term in $\tilde{R}$ of degree at least $m(m-3)$ in $\alpha$, precisely $a_{0}^{2(m-3)} \alpha^{m(m-3)}$. We study whether this term appears or not.

By Lemma 3.5, for nonzero $\alpha$ the critical points of $L_{\alpha}\left(x^{m}\right)$ are non degenerate, so $\tilde{R}\left(a_{0}=1, a_{1}=0, \ldots, a_{m}=0, \alpha=1\right) \neq 0$ and this term does appear. Choosing a polynomial $f \in \mathbb{F}_{q}[x]$ of degree $m$ amounts to choosing coefficients $a_{0}, \ldots, a_{m}$ in $\mathbb{F}_{q}$ with $a_{0} \neq 0$. Thus we can consider $\tilde{R}$ as a nonzero polynomial in $\alpha$ of degree $m(m-3)$ which has at most $m(m-3)$ roots. 
3.3. The condition (b). We use a similar strategy to prove that for almost every choice of $\alpha$ the polynomial $L_{\alpha} f$ has distinct critical values: we use an homogeneity result and we study the case of $L_{\alpha}\left(x^{m}\right)$. As it is a key point in our approach, we give equivalent conditions for $L_{\alpha}\left(x^{m}\right)$ to have distinct critical values. Recall that we work with $m \equiv 3(\bmod 4)$ and that we set $d=(m-1) / 2$.

Proposition 3.6. Let $m$ be an integer such that $m \geqslant 7$ and $m \equiv 3(\bmod 4)$.

(i) If there exists $\alpha \in \overline{\mathbb{F}}_{2}^{*}$ such that $L_{\alpha}\left(x^{m}\right)$ has distinct critical values then it holds true for any $\alpha \in \overline{\mathbb{F}}_{2}^{*}$.

(ii) Suppose that for any $\alpha \in \overline{\mathbb{F}}_{2}^{*}$ (or equivalently for $\alpha=1$ ) the polynomial $L_{\alpha}\left(x^{m}\right)$ has distinct critical values. Let $f \in \mathbb{F}_{q}[x]$ be a polynomial of degree $m$. Then $L_{\alpha} f$ has distinct critical values except for at most $(5 m-1)(m-3)(m-7) / 64$ values of $\alpha \in \overline{\mathbb{F}}_{2}$.

Proof. Let $\alpha \in \overline{\mathbb{F}}_{2}^{*}$ such that $L_{\alpha}\left(x^{m}\right)$ has distinct critical values. Now let $\alpha^{\prime} \in \overline{\mathbb{F}}_{2}^{*}$ and let us show that $L_{\alpha^{\prime}}\left(x^{m}\right)$ has distinct critical values. We use the characterization given by Lemma 3.7 ; suppose that $(\tau, \eta) \in\left(\overline{\mathbb{F}}_{2}\right)^{2}$ are such that

$$
\tau^{m-1}+\left(\tau+\alpha^{\prime}\right)^{m-1}=\eta^{m-1}+\left(\eta+\alpha^{\prime}\right)^{m-1}=0
$$

and

$$
\tau^{m}+\left(\tau+\alpha^{\prime}\right)^{m}=\eta^{m}+\left(\eta+\alpha^{\prime}\right)^{m} .
$$

Mutliply Equation (2) by $\left(\frac{\alpha}{\alpha^{\prime}}\right)^{m-1}$ and Equation (3) by $\left(\frac{\alpha}{\alpha^{\prime}}\right)^{m}$, we obtain that $\frac{\alpha}{\alpha^{\prime}} \eta \in\left\{\frac{\alpha}{\alpha^{\prime}} \tau, \frac{\alpha}{\alpha^{\prime}} \tau+\alpha\right\}$ i.e. $\eta \in\left\{\tau, \tau+\alpha^{\prime}\right\}$ which gives the result.

To prove assertion (ii) we follow the strategy of the proof of Proposition [3.2. Consider $f=\sum_{i=0}^{m} a_{m-i} x^{i} \in \mathbb{F}_{2}\left[a_{0}, \ldots, a_{m}\right][x]$ and $L_{\alpha} f=$ $\sum_{i=0}^{d} b_{d-i} x^{i} \in \mathbb{F}_{2}\left[b_{0}, \ldots, b_{d}, \alpha\right][x]$. By Lemma 3.8, when setting $N=d\left(\begin{array}{c}(d-1) / 2 \\ 2\end{array}\right)$ we can see $b_{0}^{N} \times \Pi\left(L_{\alpha} f\right)$ as a polynomial of $\mathbb{F}_{2}\left[a_{0}, \ldots, a_{m}, \alpha\right]$. Now we use the homogeneity result of Lemma 3.8 to know that this last polynomial has at most one term of degree at least $(5 d+2)\left(\begin{array}{c}(d-1) / 2 \\ 2\end{array}\right)$ in $\alpha$. Precisely, this

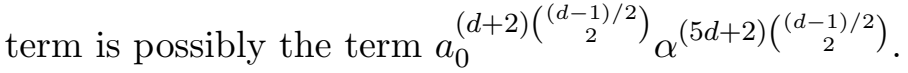

In order to know if this term appears or not, we evaluate this polynomial at $a_{0}=1$ and $a_{i}=0$ for all $i>0$ which amounts to determine if the polynomial $L_{\alpha}\left(x^{m}\right)$ has distinct critical values, which is true by hypothesis. Now fix a polynomial $f \in \mathbb{F}_{q}[x]$ of degree $m$ and see $b_{0}^{N} \times \Pi\left(L_{\alpha} f\right)$ as a polynomial of $\mathbb{F}_{2}[\alpha]$. So we know its degree and thus $L_{\alpha} f$ has distinct critical values except for at most $(5 d+2)\left(\begin{array}{c}(d-1) / 2 \\ 2\end{array}\right)$ values of $\alpha \in \overline{\mathbb{F}}_{2}$. Then we conclude using the relation between $m$ and $d$.

The following lemma gives a condition on $D_{\alpha} f$ for $L_{\alpha} f$ to have distinct critical values.

Lemma 3.7. Let $f \in \mathbb{F}_{q}[x]$. For all $\alpha \in \mathbb{F}_{q}^{*}$ the polynomial $L_{\alpha} f$ has distinct critical values if and only if for all $(\tau, \eta) \in\left(\overline{\mathbb{F}}_{2}\right)^{2},\left(D_{\alpha} f\right)^{\prime}(\tau)=\left(D_{\alpha} f\right)^{\prime}(\eta)=$ 0 and $D_{\alpha} f(\tau)=D_{\alpha} f(\eta)$ imply $\tau=\eta$ or $\tau=\eta+\alpha$. 
Proof. We have $L_{\alpha} f \circ T_{\alpha}=D_{\alpha} f$, so $\left(D_{\alpha} f\right)^{\prime}=\alpha\left(L_{\alpha} f\right)^{\prime} \circ T_{\alpha}$ where $T_{\alpha}(x)=$ $x(x+\alpha)$. The result follows noticing that $T_{\alpha}(\tau)=T_{\alpha}(\eta)$ if and only if $\tau \in\{\eta, \eta+\alpha\}$.

Lemma 3.8. Let $m \geqslant 7$ such that $m \equiv 3(\bmod 4)$ and set $N=d\left(\begin{array}{c}(d-1) / 2 \\ 2\end{array}\right)$. We consider the polynomials $f=\sum_{k=0}^{m} a_{m-k} x^{k} \in \mathbb{F}_{2}\left[a_{0}, \ldots, a_{m}\right][x]$ and $L_{\alpha} f=\sum_{k=0}^{d} b_{d-k} x^{k} \in \mathbb{F}_{2}\left[b_{0}, \ldots, b_{d}, \alpha\right][x]$. Then $b_{0}^{N} \times \Pi\left(L_{\alpha} f\right)$ is a polynomial of $\mathbb{F}_{2}\left[a_{0}, \ldots, a_{m}, \alpha\right]$ whose each term contains a product of $(d+$ $2)\left(\begin{array}{c}(d-1) / 2 \\ 2\end{array}\right)$ terms $a_{i}$. This polynomial is also homogeneous of degree $(5 d+$ $2)\left(\begin{array}{c}(d-1) / 2 \\ 2\end{array}\right)$ when considering the weight $w$ such that $w(\alpha)=1$ and $w\left(a_{i}\right)=i$.

Proof. We set $\tau_{1}, \ldots, \tau_{(d-1) / 2}$ the double roots of the polynomial $\left(L_{\alpha} f\right)^{\prime}$, and $\Pi\left(L_{\alpha} f\right)=\prod_{i \neq j}\left(L_{\alpha} f\left(\tau_{i}\right)-L_{\alpha} f\left(\tau_{j}\right)\right)$. Then we have

$$
\Pi\left(L_{\alpha} f\right)=\prod_{i<j}\left(\sum_{k=0}^{d} b_{d-k}^{2}\left(\tau_{i}^{2 k}+\tau_{j}^{2 k}\right)\right) .
$$

So $\Pi\left(L_{\alpha} f\right)$ is an homogeneous polynomial of degree $2 d\left(\begin{array}{c}(d-1) / 2 \\ 2\end{array}\right)$ when considering the weight $w$ such that $w\left(b_{i}\right)=i$ for all $i$ and $w\left(\tau_{j}\right)=1$ for all $j$. We also have that $\Pi\left(L_{\alpha} f\right) \in \mathbb{F}_{2}\left[b_{0}, \ldots, b_{d}, \tau_{1}^{2}, \ldots, \tau_{(d-1) / 2}^{2}\right]$, and each term of $\Pi\left(L_{\alpha} f\right)$ contains a product of exactly $\left(\begin{array}{c}(d-1) / 2 \\ 2\end{array}\right)$ terms $b_{i}^{2}$. Moreover, using the invariance under the action of $\mathfrak{S}_{(d-1) / 2}$ and the theorem of symmetric functions, we obtain that $\Pi\left(L_{\alpha} f\right) \in \mathbb{F}_{2}\left[b_{0}, \ldots, b_{d}, \sigma_{1}, \ldots, \sigma_{(d-1) / 2}\right]$ where $\sigma_{1}=\sum \tau_{i}^{2}, \sigma_{2}=\sum_{i<j} \tau_{i}^{2} \tau_{j}^{2}, \ldots$ Using $\left(L_{\alpha} f\right)^{\prime}=b_{0} \prod_{i=1}^{(d-1) / 2}\left(x^{2}+\tau_{i}^{2}\right)$ it follows that $\Pi\left(L_{\alpha} f\right) \in \mathbb{F}_{2}\left[b_{0}, \ldots, b_{d}, \frac{b_{2}}{b_{0}}, \frac{b_{4}}{b_{0}}, \ldots, \frac{b_{d-1}}{b_{0}}\right]$. The denominator is at worst $b_{0}^{N}$ (it happens if the $\tau_{i}$ are the only terms contributing to the degree, and if they only give rise to terms $\left.b_{2} / b_{0}\right)$. We deduce that $b_{0}^{N} \times \Pi\left(L_{\alpha} f\right)$ is a polynomial in the $b_{i}$, and that each term is a product of $(d+2)\left(\begin{array}{c}(d-1) / 2 \\ 2\end{array}\right)$ indeterminates $b_{i}$. Furthermore, it is an homogeneous polynomial of degree $2 d\left(\begin{array}{c}(d-1) / 2 \\ 2\end{array}\right)$ when considering the weight $w$ such that $w\left(b_{i}\right)=i$ for all $i$.

By Lemma 2.4, $b_{i}$ is an homogeneous polynomial of $\mathbb{F}_{2}\left[a_{0}, \ldots, a_{m}, \alpha\right]$ of degree $2 i+1$ when considering the weight $w$ such that $w\left(a_{i}\right)=i$ and $w(\alpha)=$ 1. We conclude that $b_{0}^{N} \times \Pi\left(L_{\alpha} f\right)$ is an homogeneous polynomial of degree $2 \times 2 d\left(\begin{array}{c}(d-1) / 2 \\ 2\end{array}\right)+(d+2)\left(\begin{array}{c}(d-1) / 2 \\ 2\end{array}\right)$.

Finally we reach the goal of this section: Proposition 3.2 and Proposition 3.6 enable us to bound the number of $\alpha$ such that $L_{\alpha} f$ is Morse.

Theorem 3.9. Let $m \geqslant 7$ such that $m \equiv 3(\bmod 4)$ and such that the polynomial $L_{1}\left(x^{m}\right)$ has distinct critical values. Then for all $f \in \mathbb{F}_{2^{n}}[x]$ of degree $m$ the number of elements $\alpha$ in $\mathbb{F}_{2^{n}}^{*}$ such that $L_{\alpha} f$ is Morse is at least $2^{n}-1-\frac{1}{64}(m-3)\left(5 m^{2}+28 m+7\right)$.

Proof. Let $f \in \mathbb{F}_{2^{n}}[x]$ of degree $m$ and let $\alpha \in \mathbb{F}_{2^{n}}^{*}$. The polynomial $L_{\alpha} f$ is Morse if the three conditions (a), (b) and (c) of Definition 3.1 hold. As 
$m \equiv 3(\bmod 4)$ the condition $(\mathrm{c})$ is satisfied. Indeed, $D_{\alpha} f$ has degree $m-1$ by Proposition 2.1 and thus $L_{\alpha} f$ has odd degree $(m-1) / 2$. Moreover the condition (a) fails for at most $m(m-3)$ values of $\alpha$ by Proposition 3.2 . Furthermore the condition (b) fails for at most $(5 m-1)(m-3)(m-7) / 64$ values of $\alpha$ by Proposition 3.6. Thus $L_{\alpha} f$ is not Morse for at most $m(m-$ $3)+(5 m-1)(m-3)(m-7) / 64$ values of $\alpha$.

3.4. Conditions for $L_{1}\left(x^{m}\right)$ to have distinct critical values. The condition (b) which is essential for the proofs of our main results leads by Proposition 3.6 to study for which exponents $m$ the polynomial $L_{\alpha}\left(x^{m}\right)$ has distinct critical values. By the first assertion of Proposition 3.6 we are reduced to consider the polynomial $L_{1}\left(x^{m}\right)$. Then it is natural to introduce the following set $\mathcal{M}$ and to look for practical characterizations.

Definition 3.10. Let $\mathcal{M}$ be the set of odd integers $m$ such that the polynomial $L_{1}\left(x^{m}\right)$ has distinct critical values or equivalently such that for any $\alpha \in \overline{\mathbb{F}}_{2}^{*}$ the polynomial $L_{\alpha}\left(x^{m}\right)$ has distinct critical values.

Lemma 3.7 reduces the study of the critical values of $L_{\alpha}\left(x^{m}\right)$ to the study of equations involving $D_{\alpha}\left(x^{m}\right)$ and $\left(D_{\alpha}\left(x^{m}\right)\right)^{\prime}=x^{m-1}+(x+\alpha)^{m-1}$ for odd $m$.

The following proposition enables us to have a characterization of the elements of $\mathcal{M}$ in terms of roots of unity.

Proposition 3.11. Let $m \geqslant 7$ be an odd integer. Whatever the choice of $\alpha \in \overline{\mathbb{F}}_{2}^{*}$, the polynomial $L_{\alpha}\left(x^{m}\right)$ has distinct critical values if and only if the following condition is satisfied:

for $\zeta_{1}$ and $\zeta_{2}$ in $\overline{\mathbb{F}}_{2} \backslash\{1\}$, the equalities $\zeta_{1}^{m-1}=\zeta_{2}^{m-1}=\left(\frac{1+\zeta_{1}}{1+\zeta_{2}}\right)^{m-1}=1$ imply $\zeta_{1}=\zeta_{2}$ or $\zeta_{1}=\zeta_{2}^{-1}$.

Proof. We use Lemma 3.7 to relate with the equations of Lemma 3.12, With the expressions of $x_{i}$ and $x_{j}$ obtained, we notice that $x_{i}=x_{j}+\alpha$ if and only if $\zeta_{1} \zeta_{2}=1$.

Lemma 3.12. Let $m \geqslant 7$ be an odd integer and $\alpha \in \mathbb{F}_{q}^{*}$. Two distinct elements $x_{i}$ and $x_{j}$ in $\overline{\mathbb{F}}_{2}$ satisfy

$$
\begin{gathered}
x_{i}^{m-1}=\left(x_{i}+\alpha\right)^{m-1}, x_{j}^{m-1}=\left(x_{j}+\alpha\right)^{m-1} \text { and } \\
x_{i}^{m}+\left(x_{i}+\alpha\right)^{m}=x_{j}^{m}+\left(x_{j}+\alpha\right)^{m} \quad(\diamond)
\end{gathered}
$$

if and only if $x_{i}=\frac{\zeta_{1}\left(1+\zeta_{2}\right)}{\zeta_{1}+\zeta_{2}} \alpha$ and $x_{j}=\frac{\left(1+\zeta_{2}\right)}{\zeta_{1}+\zeta_{2}} \alpha$ where $\zeta_{1}$ and $\zeta_{2}$ are two distinct elements in $\overline{\mathbb{F}}_{2} \backslash\{1\}$ such that $\zeta_{1}^{m-1}=\zeta_{2}^{m-1}=\left(\frac{1+\zeta_{1}}{1+\zeta_{2}}\right)^{m-1}=1$.

Proof. Suppose that $x_{i}$ and $x_{j}$ satisfy the first set of conditions above. We notice that they cannot be 0 neither $\alpha$, so we can set $\zeta_{1}=x_{i} / x_{j}$ and $\zeta_{2}=$ $\left(x_{i}+\alpha\right) /\left(x_{j}+\alpha\right)$. As $x_{i} \neq x_{j}$ we have $\zeta_{1} \neq \zeta_{2}$. Replacing $\left(x_{i}+\alpha\right)^{m-1}$ by $x_{i}^{m-1}$ and $\left(x_{j}+\alpha\right)^{m-1}$ by $x_{j}^{m-1}$ in $(\diamond)$ we obtain $\zeta_{1}^{m-1}=1$. Replacing 
$x_{i}^{m-1}$ by $\left(x_{i}+\alpha\right)^{m-1}$ and $x_{j}^{m-1}$ by $\left(x_{j}+\alpha\right)^{m-1}$ in $(\diamond)$ we obtain $\zeta_{2}^{m-1}=1$. Replacing $x_{i}$ by $\zeta_{1} x_{j}$ and $x_{i}+\alpha$ by $\zeta_{2}\left(x_{j}+\alpha\right)$ in the left hand side of $(\diamond)$, we obtain $\left(1+\zeta_{1}\right) x_{j}^{m}=\left(1+\zeta_{2}\right)\left(x_{j}+\alpha\right)^{m}$, so $\left(1+\zeta_{1}\right) /\left(1+\zeta_{2}\right)=\left(x_{j}+\alpha\right) / x_{j}$, and $\left(\left(1+\zeta_{1}\right) /\left(1+\zeta_{2}\right)\right)^{m-1}=1$. To obtain the claimed expressions of $x_{i}$ and $x_{j}$, one can replace $x_{j}$ by $\zeta_{1}^{-1} x_{i}$ in the equality $x_{i}+\alpha=\zeta_{2}\left(x_{j}+\alpha\right)$. The converse follows from straightforward computations.

Example 3.13. It is straightforward to see that the integers $m=2^{k}+1$ for $k \geqslant 1$ belong to $\mathcal{M}$ since 1 is the only root of $x^{2^{k}}+1$.

Remark 3.14. As a consequence of Proposition 3.11 an odd integer $m$ belongs to $\mathcal{M}$ if and only if $2(m-1)+1$ does. It implies that if an odd integer $m$ belongs to $\mathcal{M}$ then for all $k \geqslant 0$ the integer $2^{k}(m-1)+1$ does. We also notice that if an integer $m$ (not necessary odd) satisfy the condition of Proposition 3.11 then $2(m-1)+1$ is an element of $\mathcal{M}$.

Example 3.15. As the polynomial $x^{3}-1$ has exactly two roots $\zeta$ and $\zeta^{-1}$ different from the unity, we can deduce that $m=4$ satisfies the condition of Proposition 3.11. Thus according to the above remark, the integers $2^{k} 3+1$ belong to $\mathcal{M}$ for $k \geqslant 1$.

Example 3.16. Proposition 3.11 also provides us with a method to check if an odd integer $m$ belongs to $\mathcal{M}$. For a fixed odd integer $m$, write $m-1=t 2^{s}$ with $t$ odd. Hence the $(m-1)$-th roots of unity are exactly the $t$-th roots of unity in characteristic two. Consider the smallest integer $n$ such that $2^{n} \equiv 1$ $(\bmod t)$ and compute the list of the $t$-th roots of unity distinct from 1 in the field $\mathbb{F}_{2^{n}}$. Then check for $\zeta_{1}$ and $\zeta_{2}$ in this list if $\left(\frac{1+\zeta_{1}}{1+\zeta_{2}}\right)^{t}=1$ imply $\zeta_{1}=\zeta_{2}$ or $\zeta_{1}=\zeta_{2}^{-1}$ using an exhaustive method. For example using the open source computer algebra system SAGE we have determined that the only odd integers less than 200 which do not belong to $\mathcal{M}$ are $15,29,31$, $43,57,61,63,71,85,91,99,103,113,121,125,127,141,147,151,155$, $169,171,179,181,183,187$ and 197.

We give below some infinite families of good exponents.

Example 3.17. Let us prove that for any $k \geqslant 0$ the integers $m=2^{k}+2$ satisfy the conditions of Proposition 3.11. First notice that if $\zeta$ is a $(m-1)$ th root of unity then $(1+\zeta)^{2^{k}+1}=\zeta+\zeta^{-1}$. As a consequence, if $\zeta_{1}$ and $\zeta_{2}$ are two $(m-1)$-th roots of unity such that $\left(\frac{1+\zeta_{1}}{1+\zeta_{2}}\right)^{m-1}=1$ then

$$
\zeta_{2}\left(\left(1+\zeta_{1}\right)^{2^{k}+1}+\left(1+\zeta_{2}\right)^{2^{k}+1}\right)=\zeta_{2}^{2}+\left(\zeta_{1}+\zeta_{1}^{-1}\right) \zeta_{2}+1
$$

But this is equal to zero, so $\zeta_{2}$ is equal to $\zeta_{1}$ or $\zeta_{1}^{-1}$.

Example 3.18. Applying Remark 3.14 to the previous example we deduce that for any $k$ and $s$ satisfying $k \geqslant s \geqslant 1$ the integer $2^{k}+2^{s}+1$ belongs to $\mathcal{M}$. 
Example 3.19. In the case where $m=2^{k}-1$, with $k \geqslant 4$, we notice that for any choice of $\zeta_{1}$ a $\left(2^{k-1}-1\right)$-th root of unity, we also have $\left(1+\zeta_{1}\right)^{2^{k-1}-1}=1$. So any choice of a couple $\left(\zeta_{1}, \zeta_{2}\right)$ of $\left(2^{k-1}-1\right)$-th roots of unity such that $\zeta_{1} \neq$ $\zeta_{2}$ and $\zeta_{1} \zeta_{2} \neq 1$ will satisfy the hypothesis $\zeta_{1}^{m-1}=\zeta_{2}^{m-1}=\left(\frac{1+\zeta_{1}}{1+\zeta_{2}}\right)^{m-1}=1$ but will not satisfy the conclusion. In this case $L_{\alpha}\left(x^{m}\right)$ does not have distinct critical values so $m \notin \mathcal{M}$.

The following result will be our main tool to obtain infinite families of good exponents with convenient congruence. Indeed this result combined with the characterization of the set $\mathcal{M}$ given in Proposition 3.11 will provide us the families of good exponents explicited in Proposition 5.2 (iii) and exploited in Corollaries 5.4 and 5.6.

Proposition 3.20. Let $p, \ell$ be distinct primes such that $\ell \neq 2, p^{\ell-1} \not \equiv 1$ $\left(\bmod \ell^{2}\right)$ and that, if $\zeta_{1}, \zeta_{2} \neq 1$ are $\ell$-th roots of unity in characteristic $p$ such that $\left(\zeta_{1}+1\right) /\left(\zeta_{2}+1\right)$ is also a $\ell$-th root of unity, then $\zeta_{1}=\zeta_{2}$ or $\zeta_{1}=\zeta_{2}^{-1}$. Then, for any $k \geq 2$, if $\zeta_{1}, \zeta_{2} \neq 1$ are $\ell^{k}$-th roots of unity in characteristic $p$ such that $\left(\zeta_{1}+1\right) /\left(\zeta_{2}+1\right)$ is also a $\ell^{k}$-th root of unity, then $\zeta_{1}=\zeta_{2}$ or $\zeta_{1}=\zeta_{2}^{-1}$.

Proof. Induction on $k$. The case $k=1$ is the hypothesis.

Assume now that $\zeta_{1}$ have order exactly $\ell^{k}, k \geq 2$ and let $\mathbb{F}_{q}=\mathbb{F}_{p}\left(\zeta_{1}\right)$. Because we assumed that $p^{\ell-1} \not \equiv 1\left(\bmod \ell^{2}\right)$, we have that the order of $p$ $\left(\bmod \ell^{k}\right)$ is $\ell$ times the order of $p\left(\bmod \ell^{k-1}\right)$. Let $\mathbb{F}_{r}=\mathbb{F}_{p}\left(\zeta_{1}^{\ell}\right)$. It follows that $\left[\mathbb{F}_{q}: \mathbb{F}_{p}\right]=\ell\left[\mathbb{F}_{r}: \mathbb{F}_{p}\right]$. Then $q=r^{\ell}$ and the minimal polynomial of $\zeta_{1}$ over $\mathbb{F}_{r}$ is $x^{\ell}-\alpha_{1}$, where $\alpha_{1}=\zeta_{1}^{\ell}$ has order $\ell^{k-1}$. In particular $\mathrm{N} \zeta_{1}=\alpha_{1}, \operatorname{Tr} \zeta_{1}=0$ and $\mathrm{N}\left(1+\zeta_{1}\right)=1+\alpha_{1}$ where $\mathrm{N}, \operatorname{Tr}$ are respectively the norm and trace $\mathbb{F}_{q} / \mathbb{F}_{r}$, and the last equality follows by evaluating $x^{\ell}-\alpha_{1}$ at $x=-1$.

Assume first that $\zeta_{2}$ have order exactly $\ell^{k}$ also and that $\zeta_{3}=\left(\zeta_{1}+\right.$ $1) /\left(\zeta_{2}+1\right)$ is also a $\ell^{k}$-th root of unity and write $\zeta_{i}^{\ell}=\alpha_{i}, i=2,3$ so the $\alpha_{i}$ are $\ell^{k-1}$-th roots of unity. As before, we get that $\mathrm{N} \zeta_{i}=\alpha_{i}, i=2,3$ and that $\mathrm{N}\left(1+\zeta_{2}\right)=1+\alpha_{2}$. Taking norms, we get $\alpha_{3}=\left(\alpha_{1}+1\right) /\left(\alpha_{2}+1\right)$, so by induction we get that $\alpha_{1}=\alpha_{2}$ or $\alpha_{1}=\alpha_{2}^{-1}$.

If $\alpha_{1}=\alpha_{2}$, then $\alpha_{3}=1$ and either $\zeta_{1}=\zeta_{2}$ as we wanted or $\zeta_{1}=\omega \zeta_{2}$ with $\omega$ of order $\ell$. In the latter case we get $\left(1+\omega \zeta_{2}\right) /\left(1+\zeta_{2}\right)=\omega^{j}$ for some $j=0,1, \ldots, \ell-1$. If $j \neq 1$, we can solve the equation for $\zeta_{2}$ and get $\zeta_{2} \in \mathbb{F}_{p}(\omega)$ which is a contradiction. If $j=1$ we get $\omega=1$, also a contradiction.

If $\alpha_{1}=\alpha_{2}^{-1}$, then $\alpha_{3}=\alpha_{1}$ and either $\zeta_{1}=\zeta_{2}^{-1}$ as we wanted or $\zeta_{1}=\omega \zeta_{2}^{-1}$ with $\omega$ of order $\ell$. In the latter case we get $\left(1+\zeta_{1}\right) /\left(1+\omega \zeta_{1}^{-1}\right)=\omega^{j} \zeta_{1}$ for some $j=0,1, \ldots, \ell-1$. This gives, for $j \neq 0, \zeta_{1} \in \mathbb{F}_{p}(\omega)$ which is a contradiction. For $j=0$, this gives $\omega=1$, also a contradiction.

Finally, assume that $\zeta_{2}$ have order smaller than $\ell^{k}$, so $\zeta_{2} \in \mathbb{F}_{r}$. We write our equation as $\left(\zeta_{1}+1\right)=\zeta_{3}\left(\zeta_{2}+1\right)$. First note that $\zeta_{3}$ cannot be in $\mathbb{F}_{r}$, 
since $\zeta_{1}$ is not in $\mathbb{F}_{r}$, so $\operatorname{Tr} \zeta_{1}=\operatorname{Tr} \zeta_{3}=0$, so taking trace of our equation gives $1=0\left(\zeta_{2}+1\right)=0$, contradiction.

Example 3.21. We verified by computer calculation that the hypothesis of this proposition holds when $p=2$ and $\ell<200$ except for $\ell=$ $7,31,73,89,127$. For example the case $\ell=3$ follows from Example 3.15, These computations will enable us to exhibit the examples of Corollaries 5.4 and 5.6 .

\section{Regular extensions}

Let $n$ be an integer $\geqslant 1$ and set $q=2^{n}$. Let $t$ be an element transcendental over $\mathbb{F}_{q}$ and $K$ an extension field of $\mathbb{F}_{q}(t)$. Recall that the extension $K / \mathbb{F}_{q}(t)$ is said to be regular if it is separable and if $\mathbb{F}_{q}$ is algebraically closed in $K$ i.e. $\mathbb{F}_{q}^{K}=\mathbb{F}_{q}$ where $\mathbb{F}_{q}^{K}$ is the algebraic closure of $\mathbb{F}_{q}$ in $K$.

Let $\alpha \in \mathbb{F}_{q}^{*}$, let $m$ be an integer and $d=(m-1) / 2$ if $m$ is odd and $d=(m-2) / 2$ if $m$ is even. Fix $f \in \mathbb{F}_{q}[x]$ a polynomial of degree $m$ such that the associated polynomial $L_{\alpha} f$ has degree exactly $d$. Furthermore, we suppose that $d$ is odd which is equivalent to say that $m \equiv 0(\bmod 4)$ or $m \equiv 3(\bmod 4)$.

4.1. First floor: monodromy. We consider the arithmetic monodromy group $G$ of the polynomial $L_{\alpha} f$. It is the Galois group of the extension $F / \mathbb{F}_{q}(t)$ where $F$ is the splitting field of the polynomial $L_{\alpha} f(x)-t$ over the field $\mathbb{F}_{q}(t)$. Consider also $\bar{G}:=\operatorname{Gal}\left(F / \mathbb{F}_{q}^{F}(t)\right)$ the geometric monodromy group of $L_{\alpha} f$. The groups $G$ and $\bar{G}$ are transitive subgroups of the symmetric group $\mathfrak{S}_{d}$ and $\bar{G} \triangleleft G$.

Proposition 4.1. Let $f \in \mathbb{F}_{q}[x]$ be a polynomial such that the associated polynomial $L_{\alpha} f$ is Morse and has (odd) degree $d$.

(i) Let $u$ be a root of $L_{\alpha} f(x)-t$ in $F$. Then, for each place $\wp$ of $F$ above the place $\infty$ at infinity of $\mathbb{F}_{q}(t)$, we have that $u$ has a simple pole at $\wp$.

(ii) The group $\operatorname{Gal}\left(F / \mathbb{F}_{q}(t)\right)$ is the full symmetric group $\mathfrak{S}_{d}$ and the extension $F / \mathbb{F}_{q}(t)$ is regular.

Proof. If $v_{\wp}$ is the valuation at the place $\wp$, we have $v_{\wp}\left(L_{\alpha} f(u)\right)=v_{\wp}(t)$ and by definition of the ramification index $e(\wp \mid \infty)$ we have $v_{\wp}(t)=e(\wp \mid \infty) v_{\infty}(t)=$ $-e(\wp \mid \infty)$. Since $d$ is supposed to be odd, it is prime to the characteristic of $\mathbb{F}_{q}(t)$, and then, by the proof of Theorem 4.4.5 of [9], we have $e(\wp \mid \infty)=d$. Hence, we obtain $v_{\wp}\left(L_{\alpha} f(u)\right)=-d$, which implies that $v_{\wp}(u)=-1$ and thus $u$ has a simple pole at $\wp$.

The analogue of the Hilbert theorem given by Serre in Theorem 4.4.5 of [9] and detailled in even characteristic in the appendix of Geyer in 5] gives that the geometric monodromy group $\operatorname{Gal}\left(F / \mathbb{F}_{q}^{F}(t)\right)$ of $L_{\alpha} f$ is the symmetric group $\mathfrak{S}_{d}$. But it is contained in the arithmetic monodromy 
$\operatorname{group} \operatorname{Gal}\left(F / \mathbb{F}_{q}(t)\right)$ which is also a subgroup of $\mathfrak{S}_{d}$. So they are equal and $\mathbb{F}_{q}^{F}=\mathbb{F}_{q}$.

A consequence of the first part of the previous proposition is that $L_{\alpha} f(x)-$ $t$ has only simple roots; let us call them $u_{0}, \ldots, u_{d-1}$.

4.2. Second floor. Let $x_{i}$ such that $x_{i}^{2}+\alpha x_{i}=u_{i}$. Hence we have $D_{\alpha} f\left(x_{i}\right)=$ $t$. Consider $\Omega=\mathbb{F}_{q}\left(x_{0}, \ldots, x_{d-1}\right)$ the compositum of the fields $F\left(x_{i}\right)$ and $\mathbb{F}_{q}^{\Omega} F$ the compositum of $F$ and $\mathbb{F}_{q}^{\Omega}$. Let $\Gamma=\operatorname{Gal}(\Omega / F)$ and $\bar{\Gamma}=\operatorname{Gal}\left(\Omega / \mathbb{F}_{q}^{\Omega} F\right)$.

The following statement appears in $[10$.

Lemma 4.2. Suppose that $L_{\alpha} f$ is Morse and has degree $d$. If $J \subset\{0, \ldots, d-$ $1\}$ is neither empty nor the whole set then $\sum_{j \in J} u_{j}$ has a pole at a place of $F$ over the place $\infty$ of $\mathbb{F}_{q}(t)$.

Proof. To obtain a contradiction suppose that $J \subset\{0, \ldots, d-1\}$ is such that $j_{0} \in J$ whereas $j_{1} \in\{0, \ldots, d-1\} \backslash J$. Suppose also that $\sum_{j \in J} u_{j}$ has no pole in places above $\infty$. Then it has no pole at all, and so it is constant. Recall that $\mathrm{Gal}\left(F / \mathbb{F}_{q}(t)\right)$ is $\mathfrak{S}_{d}$ by Proposition 4.1. Applying to $\sum_{j \in J} u_{j}$ the automorphism corresponding to the transposition $\left(j_{0} j_{1}\right) \in \mathfrak{S}_{d}$ one obtains $\sum_{j \in J \backslash\left\{j_{0}\right\}} u_{j}+u_{j_{0}}=\sum_{j \in J \backslash\left\{j_{0}\right\}} u_{j}+u_{j_{1}}$, which leads to $u_{j_{0}}=u_{j_{1}}$, a contradiction.

Lemma 4.3. Suppose that $L_{\alpha} f$ is Morse and has degree d. Let $\widetilde{F}$ be $F$ or $\mathbb{F}_{q}^{\Omega} F$. Let $J$ be a non-empty subset of $\{0, \ldots, d-1\}$ different from $\{0, \ldots, d-$ $1\}$. Then

$$
\sum_{j \in J} x_{j} \notin \widetilde{F}
$$

Proof. To obtain a contradiction, suppose that $\sum_{j \in J} x_{j} \in \widetilde{F}$. By Lemma 4.2 we know that there exists a place $\wp$ of $F$ above $\infty$ such that $\sum_{j \in J} u_{j}$ has a pole at $\wp$. Moreover, this pole is simple as for all $j \in\{0, \ldots, d-1\}$ the root $u_{j}$ has a simple pole by Proposition 4.1. Now consider $A=\left(\sum_{j \in J} x_{j}\right)$ and $B=\left(\sum_{j \in J} x_{j}+\alpha\right)$. If $A$ (and thus $B$ ) belongs to $\widetilde{F}$, one can consider the valuation of $A$ and $B$ at $\wp$. As $A . B=\sum_{j \in J} u_{j}$ it follows that either $A$ or $B$ has a pole. Since $A$ and $B$ differ from a constant, $A$ has a pole if and only if $B$ has a pole. So both have a pole and the order of multiplicity is the same. Then we obtain $2 v_{\wp}(A)=-1$, a contradiction.

Lemma 4.4. Let $k\left(x_{1}\right)$ and $k\left(x_{2}\right)$ be two Artin-Schreier extensions of a field $k$ of characteristic 2. Suppose that $x_{i}^{2}+\alpha x_{i}=w_{i}$ with $\alpha$ and $w_{i}$ in $k^{*}$. Then $k\left(x_{1}\right)=k\left(x_{2}\right)$ if and only if $x_{1}+x_{2} \in k$.

Moreover if $x_{1}+x_{2} \notin k$ then $k\left(x_{1}, x_{2}\right)$ is a degree 4 extension of $k$ and the three fields lying between $k$ and $k\left(x_{1}, x_{2}\right)$ are those of the following diagram. 


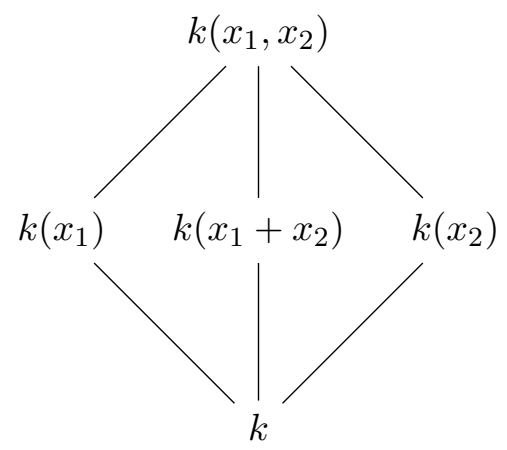

Proof. For the first assertion, see the proof of Lemma 4.1 in [1. In the case where $x_{1}+x_{2} \notin k$, we can use $\left[k\left(x_{1}\right)\left(x_{2}\right): k\left(x_{1}\right)\right]=2$ to prove $\left[k\left(x_{1}, x_{2}\right)\right.$ : $k]=4$. We deduce that $\operatorname{Gal}\left(k\left(x_{1}, x_{2}\right) / k\right)=(\mathbb{Z} / 2 \mathbb{Z})^{2}$. The field $k\left(x_{1}+x_{2}\right)$ is a subextension since $x_{1}+x_{2}$ is a root of $x^{2}+\alpha x=w_{1}+w_{2}$. It remains to prove that $k\left(x_{1}+x_{2}\right)$ is different from $k\left(x_{1}\right)$ (and $k\left(x_{2}\right)$ ). According to the first statement of the lemma, it is sufficient to check that $x_{1}+\left(x_{1}+x_{2}\right) \notin k$.

Proposition 4.5. Suppose that $L_{\alpha} f$ is Morse and has degree d. Let $\widetilde{F}$ be $F$ or $\mathbb{F}_{q}^{\Omega} F$. Let $r$ be an integer such that $0 \leqslant r \leqslant d-2$. Then

(i) the field $\widetilde{F}\left(x_{0}, \ldots, x_{r}\right)$ is an extension of order $2^{r+1}$ of $\widetilde{F}$,

(ii) the Galois group $\mathrm{Gal}\left(\widetilde{F}\left(x_{0}, \ldots, x_{r}\right) / \widetilde{F}\right)$ is $(\mathbb{Z} / 2 \mathbb{Z})^{r+1}$ and

(iii) there are $2^{r+1}-1$ quadratic extensions of $\widetilde{F}$ between $\widetilde{F}$ and $\widetilde{F}\left(x_{0}, \ldots, x_{r}\right)$.

Namely, these extensions are the extensions $\widetilde{F}\left(\sum_{j \in J} x_{j}\right)$ with nonempty $J \subset\{0, \ldots, r\}$.

Proof. We proceed by induction. The case $r=0$ is trivial and the case $r=1$ is given by Lemma 4.4. Assuming that the proposition holds for $r-1$, with $1 \leqslant r \leqslant d-2$, we will prove it for $r$. The main idea is to consider the extensions of the following diagram

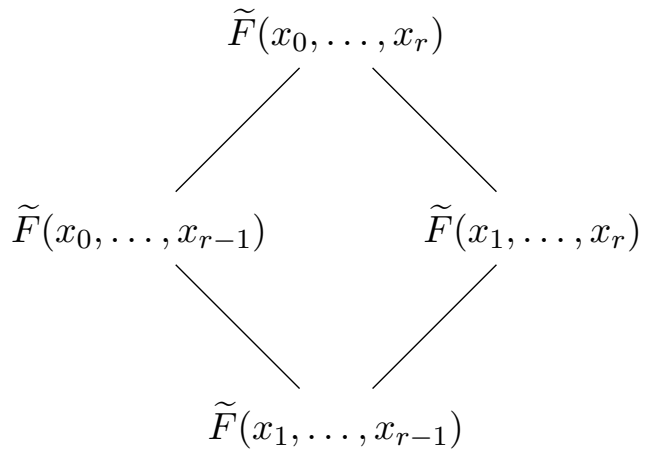

and to apply Lemma 4.4. We first prove that $x_{0}+x_{r} \notin \widetilde{F}\left(x_{1}, \ldots, x_{r-1}\right)$. Otherwise we would have the quadratic extension $\widetilde{F}\left(x_{0}+x_{r}\right)$ between $\widetilde{F}$ and $\widetilde{F}\left(x_{1}, \ldots, x_{r-1}\right)$. By the induction hypothesis, there would exist $J \subset$ 
$\{1, \ldots, r-1\}$ such that $\widetilde{F}\left(x_{0}+x_{r}\right)=\widetilde{F}\left(\sum_{j \in J} x_{j}\right)$. By Lemma 4.4 again we would have $x_{0}+x_{r}+\sum_{j \in J} x_{j} \in \widetilde{F}$ and then a contradiction with Lemma4.3, Then we can apply the conclusions of Lemma 4.4 with $k=\widetilde{F}\left(x_{1}, \ldots, x_{r-1}\right)$ to obtain that $\widetilde{F}\left(x_{0}, \ldots, x_{r}\right)$ is a quadratic extension of both $\widetilde{F}\left(x_{1}, \ldots, x_{r}\right)$ and $\widetilde{F}\left(x_{0}, \ldots, x_{r-1}\right)$. It follows that $\left[\widetilde{F}\left(x_{0}, \ldots, x_{r}\right): \widetilde{F}\right]=2^{r+1}$.

Furthermore, we can define $2^{r+1}$ different $\widetilde{F}$-automorphisms of $\widetilde{F}\left(x_{0}, \ldots, x_{r}\right)$ by sending $x_{i}$ to $x_{i}$ or to $x_{i}+\alpha$. So, all the elements of the Galois group $\operatorname{Gal}\left(\widetilde{F}\left(x_{0}, \ldots, x_{r}\right) / \widetilde{F}\right)$ have order dividing 2 thus this group is certainly $(\mathbb{Z} / 2 \mathbb{Z})^{r+1}$.

For any non-empty subset $J \subset\{0, \ldots, r\}$ we see that $\sum_{j \in J} x_{j}$ is a root of $x^{2}+\alpha x=\sum_{j \in J} u_{j}$, and we know from Lemma 4.3 that $\sum_{j \in J} x_{j} \notin \widetilde{F}$. We obtain this way $2^{r+1}-1$ different quadratic extensions between $\widetilde{F}$ and $\widetilde{F}\left(x_{0}, \ldots, x_{r}\right)$. Indeed, we can show that these extensions are different. If $\widetilde{F}\left(\sum_{j \in J_{1}} x_{j}\right)=\widetilde{F}\left(\sum_{j \in J_{2}} x_{j}\right)$ then $\sum_{j \in J_{1}} x_{j}+\sum_{j \in J_{2}} x_{j} \in \widetilde{F}$ which leads to $J_{1}=J_{2}$ using Lemma 4.3. Finally, these $2^{r+1}-1$ quadratic extensions are the only ones. Indeed, the quadratic extensions between $\widetilde{F}$ and $\widetilde{F}\left(x_{0}, \ldots, x_{r}\right)$ are in correspondence with the subgroups of $(\mathbb{Z} / 2 \mathbb{Z})^{r+1}$ of index 2 . These subgroups are the hyperplanes of $(\mathbb{Z} / 2 \mathbb{Z})^{r+1}$ and one can count $2^{r+1}-1$ of them.

Proposition 4.6. Suppose that $L_{\alpha} f=\sum_{k=0}^{d} b_{d-k} x^{k}$ is Morse and has degree d. Let $\widetilde{F}$ be $F$ or $F \mathbb{F}_{q}^{\Omega}$. If there exists $x \in \mathbb{F}_{q}$ such that $x^{2}+\alpha x=b_{1} / b_{0}$ then $\operatorname{Gal}\left(\widetilde{F}\left(x_{0}, \ldots, x_{d-1}\right) / \widetilde{F}\right)$ is $(\mathbb{Z} / 2 \mathbb{Z})^{d-1}$ and thus the extensions $\Omega / F$ and $\Omega / \mathbb{F}_{q}(t)$ are regular.

Proof. As Proposition 4.5 already gives Gal $\left(\widetilde{F}\left(x_{0}, \ldots, x_{d-2}\right) / \widetilde{F}\right)=(\mathbb{Z} / 2 \mathbb{Z})^{d-1}$, it remains to study the extension $\widetilde{F}\left(x_{0}, \ldots, x_{d-1}\right) / \widetilde{F}\left(x_{0}, \ldots, x_{d-2}\right)$.

Using $\sum_{i=0}^{d-1} u_{i}=b_{1} / b_{0}$ and the linearity of $x \mapsto x^{2}+\alpha x$, we see that in any case the equation $x^{2}+\alpha x=b_{1} / b_{0}$ has two solutions in $\overline{\mathbb{F}}_{q}$, namely $\sum_{i=0}^{d-1} x_{i}$ and $\alpha+\sum_{i=0}^{d-1} x_{i}$. With our hypothesis we deduce that $\sum_{i=0}^{d-1} x_{i} \in \mathbb{F}_{q}$ hence $\widetilde{F}\left(x_{0}, \ldots, x_{d-1}\right)=\widetilde{F}\left(x_{0}, \ldots, x_{d-2}\right)$ and the result about the Galois group follows. Thus we have proved that $\Gamma=\bar{\Gamma}$ and then $\Omega / F$ is regular. Proposition 4.1 shows that the extension $F / \mathbb{F}_{q}(t)$ is regular, hence we deduce the regularity of the extension $\Omega / \mathbb{F}_{q}(t)$.

\section{MAin Results}

The main ingredient of the proof of our main results is the Chebotarev density theorem. The next proposition summarizes its contribution in our context. 
Proposition 5.1. Let $m \geqslant 7$ be an integer such that $m \equiv 3(\bmod 4)$. Then there exists an integer $N$ depending only on $m$ such that for all $n \geqslant N$, if we set $q=2^{n}$, for all $f \in \mathbb{F}_{q}[x]$ of degree $m$, and for all $\alpha$ in $\mathbb{F}_{q}^{*}$ such that the extension $\Omega / \mathbb{F}_{q}(t)$ is regular, there exists $\beta \in \mathbb{F}_{q}$ such that the polynomial $D_{\alpha} f(x)+\beta$ splits in $\mathbb{F}_{q}[x]$ with no repeated factors.

Proof. As $m \equiv 3(\bmod 4)$, by Proposition 2.1 the polynomial $L_{\alpha} f$ has degree exactly $d=(m-1) / 2$, which is odd by our hypothesis on $m$, and thus $F / \mathbb{F}_{q}(t)$ is separable. Since the extension $\Omega / F$ is also separable we obtain that $\Omega / \mathbb{F}_{q}(t)$ is separable and thus Galois.

Since the extension $\Omega / \mathbb{F}_{q}(t)$ is supposed to be regular, by an application of the Chebotarev theorem (see Theorem 1 in [3] which is deduced from Proposition 4.6.8 in [8]) the number $N(S)$ of places $v$ of $\mathbb{F}_{q}(t)$ of degree 1 unramified in $\Omega$ and such that the Artin symbol $\left(\frac{\Omega / \mathbb{F}_{q}(t)}{v}\right)$ is equal to the conjugacy class of $\operatorname{Gal}\left(\Omega / \mathbb{F}_{q}(t)\right)$ consisting of the identity element satisfies

$$
N(S) \geqslant \frac{q}{d_{\Omega}}-2\left(\left(1+\frac{g_{\Omega}}{d_{\Omega}}\right) q^{1 / 2}+q^{1 / 4}+1+\frac{g_{\Omega}}{d_{\Omega}}\right)
$$

where $d_{\Omega}:=\left[\Omega: \mathbb{F}_{q}(t)\right]$ and $g_{\Omega}$ is the genus of $\Omega$.

But we have seen that $G=\operatorname{Gal}\left(F / \mathbb{F}_{q}(t)\right)$ is a subgroup of $\mathfrak{S}_{d}$ and $\Gamma=$ $\operatorname{Gal}(\Omega / F)$ is a group of order bounded by $2^{d}$, thus we have $d_{\Omega} \leqslant d ! 2^{d}$. Moreover, one can obtain an upper bound on $g_{\Omega}$ depending only on $d$ using Lemma 14 of [7] to get that: $g_{\Omega} \leqslant \frac{1}{2}\left(\operatorname{deg} D_{\alpha} f-3\right) d_{\Omega}+1$ i.e.

$$
g_{\Omega} \leqslant\left(d ! 2^{d}\right) \times(d-3 / 2)+1 .
$$

Then if $q$ is sufficiently large we will have $N(S) \geqslant 1$ which concludes the proof.

Since the methods of our proofs need the degree $m$ of the polynomials to belong to the set $\mathcal{M}$ defined in Definition 3.10, we sum up some infinite subsets of $\mathcal{M}$ we have pointed out in Subsection 3.4.

Proposition 5.2. The following integers $m$ belong to the set $\mathcal{M}$ :

(i) $m=2^{k}+1$ for $k \geqslant 1$.

(ii) $m=2^{k}+2^{s}+1$ for $k \geqslant s \geqslant 1$.

(iii) $m=2^{s} \ell^{k}+1$ for $k \geqslant 1, s \geqslant 1$ and for $\ell$ an odd prime such that $2^{\ell-1} \not \equiv 1\left(\bmod \ell^{2}\right)$ and such that $m^{\prime}:=\ell+1$ satisfy the condition of Proposition 3.11.

Proof. The first two assertions are proved respectively in Example 3.13 and 3.18. If $\ell$ satisfy the hypothesis (iii) then Proposition 3.20 in the case of characteristic two tells us that $\ell^{k}+1$ also satisfy the condition of Proposition 3.11. Now use Remark 3.14 to have that $2^{s} \ell^{k}+1$ satisfy the condition of Proposition 3.11. For $s \geqslant 1$ it is odd and so it belongs to $\mathcal{M}$. 
Now we can state and prove our main results which establish for some polynomials $f$ the maximality of the differential uniformity $\delta(f)$ defined in Section 1 by $\delta(f)=\max _{(\alpha, \beta) \in \mathbb{F}_{q}^{*} \times \mathbb{F}_{q}} \sharp\left\{x \in \mathbb{F}_{q} \mid f(x+\alpha)+f(x)=\beta\right\}$.

Theorem 5.3. Let $m \in \mathcal{M}$ such that $m \equiv 7(\bmod 8)$. Then for $n$ sufficiently large, for all polynomials $f \in \mathbb{F}_{2^{n}}[x]$ of degree $m$ we have $\delta(f)=$ $m-1$.

Proof. We fix $m \in \mathcal{M}$ such that $m \equiv 7(\bmod 8)$. Let us prove that for $n$ sufficiently large and for any polynomial $f=\sum_{i=0}^{m} a_{m-i} x^{i}$ in $\mathbb{F}_{2^{n}}[x]$ of degree $m$, there exists $\alpha$ in $\mathbb{F}_{2^{n}}^{*}$ such that:

$-L_{\alpha} f$ is Morse

- the equation $x^{2}+\alpha x=\frac{b_{1}}{b_{0}}$ has a solution in $\mathbb{F}_{2^{n}}$, where $L_{\alpha} f=$ $\sum_{i=0}^{d} b_{d-i} x^{i}$.

By Theorem [3.9, for all $f \in \mathbb{F}_{2^{n}}[x]$ of degree $m$, the number of elements $\alpha$ in $\mathbb{F}_{2^{n}}^{*}$ such that $L_{\alpha} f$ is Morse is at least $2^{n}-\frac{1}{64}(m-3)\left(5 m^{2}+28 m+7\right)$.

Moreover, by the Hilbert' 90 Theorem, the equation $x^{2}+\alpha x=\frac{b_{1}}{b_{0}}$ has a solution in $\mathbb{F}_{2^{n}}$ if and only if $\operatorname{Tr}_{\mathbb{F}_{2^{n}} / \mathbb{F}_{2}}\left(\frac{b_{1}}{b_{0} \alpha^{2}}\right)=0$. By Lemma 2.5 it is equivalent to $\operatorname{Tr}_{\mathbb{F}_{2^{n}} / \mathbb{F}_{2}}\left(\frac{a_{1}^{2}+a_{0} a_{2}}{a_{0}^{2} \alpha^{2}}\right)=0$. In the case where $a_{1}^{2}+a_{0} a_{2}=0$ every choice of $\alpha \in \mathbb{F}_{2^{n}}^{*}$ is convenient. Otherwise the map sending $\alpha$ to $\frac{a_{1}^{2}+a_{0} a_{2}}{a_{0}^{2} \alpha^{2}}$ is a permutation of $\mathbb{F}_{2^{n}}^{*}$ and then $2^{n-1}-1$ values of $\alpha$ are convenient.

Hence as soon as $2^{n-1}>\frac{1}{64}(m-3)\left(5 m^{2}+28 m+7\right)+1$ we will have for any $f \in \mathbb{F}_{2^{n}}[x]$ of degree $m$ the existence of $\alpha$ in $\mathbb{F}_{2^{n}}^{*}$ satisfying the two conditions. Now, these conditions imply by Proposition 4.6 that the extension $\Omega / \mathbb{F}_{2^{n}}(t)$ is regular.

Finally we can apply Proposition 5.1 to obtain, for $n$ sufficiently large depending only on $m$, the existence of $\beta \in \mathbb{F}_{2^{n}}$ such that the polynomial $D_{\alpha} f(x)+\beta$ splits in $\mathbb{F}_{2^{n}}[x]$ with no repeated factors. Then $\delta(f)=m-1$.

To be concrete, using Proposition 5.2, the computations of Example 3.21 and taking into account the congruences of $m$ we present in the following corollary some families of infinitely many integers for which Theorems 5.3 holds.

Corollary 5.4. Let $\ell$ be a prime congruent to 3 modulo 4 such that $2^{\ell-1} \not \equiv 1$ $\left(\bmod \ell^{2}\right)$ and $\ell+1$ satisfy the condition of Proposition 3.11 (for example, $\ell \in$ $\{3,11,19,23,43,47,59,67,71,79,83,103,107,131,139,151,163,167,179,191$, $199 \ldots\})$. Set $m=2 \ell^{2 k+1}+1$ with $k \geqslant 0$. Then for $n$ sufficiently large, for all polynomials $f \in \mathbb{F}_{2^{n}}[x]$ of degree $m$ we have $\delta(f)=m-1$.

When $m$ is congruent to 3 modulo 8 , we also obtain some results but we have conditions on the parity of $n$ or we have to remove some polynomials. 
Theorem 5.5. Let $m \in \mathcal{M}$ such that $m \geqslant 7$ and $m \equiv 3(\bmod 8)$.

(i) For $n$ even and sufficiently large and for all polynomials $f \in \mathbb{F}_{2^{n}}[x]$ of degree $m$ we have $\delta(f)=m-1$.

(ii) For $n$ sufficiently large and for all polynomials $f=\sum_{i=0}^{m} a_{m-i} x^{i}$ in $\mathbb{F}_{2^{n}}[x]$ of degree $m$ such that $a_{1}^{2}+a_{0} a_{2} \neq 0$, we have $\delta(f)=m-1$.

Proof. The proof is similar as the one of Theorem 5.3. The main difference comes from the expression of $b_{1} / b_{0}$ when $m \equiv 3(\bmod 8)$. According to Lemma 2.5, we have $\operatorname{Tr}_{\mathbb{F}_{2^{n}} / \mathbb{F}_{2}}\left(\frac{b_{1}}{b_{0} \alpha^{2}}\right)=0$ if and only if $\operatorname{Tr}_{\mathbb{F}_{2^{n}} / \mathbb{F}_{2}}\left(\frac{a_{1}^{2}+a_{0} a_{2}}{a_{0}^{2} \alpha^{2}}\right)=$ $n$. The arguments of the above proof apply except when $a_{1}^{2}+a_{0} a_{2}=0$ and $n$ is odd.

We remark that one could not expect better in the case where $m \equiv 3$ $(\bmod 8), a_{1}^{2}+a_{0} a_{2}=0$ and $n$ odd since Theorem 2 (iii) of [10] gives that $\delta(f)<m-1$ in this case.

Again using Proposition 5.2 and the computations of Example 3.21 we obtain the following corollary.

Corollary 5.6. Let $\ell$ be an odd prime such that $2^{\ell-1} \not \equiv 1\left(\bmod \ell^{2}\right)$ and $\ell+1$ satisfy the condition of Proposition 3.11.

(i) If $\ell \equiv 1(\bmod 8)$ then Theorem 5.5 holds for the integers $m=2 \ell^{k}+1$ with $k \geqslant 1$ (for example if $\ell \in\{17,41,97,113,137,193, \ldots\}$ ).

(ii) If $\ell \equiv 7(\bmod 8)$ then Theorem 5.5 holds for the integers $m=$ $2 \ell^{2 k+1}+1$ with $k \geqslant 0$ (for example if $\ell \in\{23,47,71,79,103,151,167$, $191,199, \ldots\})$.

Finally, we prove Conjecture 1.1 when $m \equiv 7(\bmod 8)$.

Theorem 5.7. For a given integer $m \in \mathcal{M}$ such that $m \equiv 7(\bmod 8)$, there exists $\varepsilon_{m}>0$ such that for all sufficiently large $n$, if $f$ is a polynomial of degree $m$ over $\mathbb{F}_{2^{n}}$, for at least $\varepsilon_{m} 2^{2 n}$ values of $(\alpha, \beta) \in \mathbb{F}_{2^{n}}^{*} \times \mathbb{F}_{2^{n}}$ we have $\sharp\left\{x \in \mathbb{F}_{q} \mid f(x+\alpha)+f(x)=\beta\right\}=\delta(f)=m-1$.

Proof. We follow the strategy described in the proofs above. The point is to give lower bounds for the number of choices of $\alpha$ and $\beta$. We have shown the existence of a polynomial $P$ of degree 3 such that for any $n$ and any $f \in \mathbb{F}_{2^{n}}[x]$ there exist at least $2^{n-1}+P(m)$ elements $\alpha$ such that the extension $\Omega / \mathbb{F}_{2^{n}}(t)$ is regular (see the proof of Theorem 5.3). Thus for any $\gamma_{m}<1 / 2$, for $n$ sufficiently large, there exists $\gamma_{m} 2^{n}$ suitable choices of $\alpha$. For such a choice of $\alpha$, the Chebotarev theorem used in the proof of Proposition 5.1 guarantees the existence of $\frac{1}{d ! 2^{d}} 2^{n}+Q\left(2^{n / 4}\right)$ elements $\beta$ such that $D_{\alpha} f(x)+\beta$ has $\delta(f)$ solutions where $Q$ is a polynomial of degree 2 . Thus for any $\gamma_{m}^{\prime}<1 / d ! 2^{d}$, for $n$ sufficiently large, there exist $2^{n} \gamma_{m}^{\prime}$ suitable choices of $\beta$. Hence we obtain the result for any $\varepsilon_{m}<1 / d ! 2^{d+1}$.

Remark that the proof of Theorem 5.7 provides explicit values of $\varepsilon_{m}$, namely any $\varepsilon_{m}$ between 0 and $1 / d ! 2^{d+1}$ with $d=\frac{m-1}{2}$. Remark also that, 
in the case where $m \equiv 3(\bmod 8)$, the same strategy leads to a proof of an analogue of this theorem for polynomials $f$ such that $a_{1}^{2}+a_{0} a_{2} \neq 0$ or a proof of another analogue for even $n$.

Acknowledgements: The third author would like to thank the I2M and CIRM for support in connection with a number of visits to Luminy and the Simons Foundation for financial support under grant \#234591.

Moreover, the authors thank the referee for valuable comments.

\section{REFERENCES}

[1] Yves Aubry and Fabien Herbaut. Differential uniformity and second order derivatives for generic polynomials, J. Pure Appl. Algebra 222 (2018), no. 5, 1095-1110.

[2] Eli Biham and Adi Shamir. Differential cryptanalysis of DES-like cryptosystems. $J$. Cryptology, 4(1):3-72, 1991.

[3] Pierre-Alain Fouque and Mehdi Tibouchi. Estimating the size of the image of deterministic hash functions to elliptic curves. In Progress in Cryptology - Latincrypt 2010, volume 6212 of Lecture Notes in Computer Science, pages 81-91, 2010.

[4] Andrew Granville. Arithmetic properties of binomial coefficients. I. Binomial coefficients modulo prime powers, CMS Conf. Proc., 20 Amer. Math. Soc., Providence, RI, 222 (2018), 1997.

[5] Moshe Jarden and Aharon Razon. Skolem density problems over large Galois extensions of global fields. In Hilbert's tenth problem: relations with arithmetic and algebraic geometry (Ghent, 1999), volume 270 of Contemp. Math., pages 213-235. Amer. Math. Soc., Providence, RI, 2000. With an appendix by Wulf-Dieter Geyer.

[6] Kaisa Nyberg. Differentially uniform mappings for cryptography. In Advances in cryptology-Eurocrypt'93, pages 55-64. Springer, 1994.

[7] Paul Pollack. Simultaneous prime specializations of polynomials over finite fields. Proc. London Math. Soc., 97(3):545-567, 2008.

[8] Michael Rosen. Number theory in function fields. New York, NY: Springer, 2002.

[9] Jean-Pierre Serre. Topics in Galois theory. CRC Press, 2007.

[10] José Felipe Voloch. Symmetric cryptography and algebraic curves. In Proceedings of the First SAGA Conference, Papeete, France. World Scientific, 2007.

(Aubry) Institut de Mathématiques de Toulon - IMATH, Université de Toulon, FRANCE

(Aubry) Institut de Mathématiques de Marseille - I2M, Aix Marseille Univ, CNRS, Centrale Marseille, France

E-mail address: yves.aubry@univ-tln.fr

(Herbaut) ESPE Nice-Toulon, Université de Nice Sophia-Antipolis, France

(Herbaut) Institut de Mathématiques de Toulon - IMATH, Université de Toulon, France

E-mail address: fabien.herbaut@unice.fr

(Voloch) School of Mathematics and Statistics, University of Canterbury, Private Bag 4800, Christchurch 8140, New Zealand

E-mail address: felipe.voloch@canterbury.ac.nz

$U R L:$ http://www.math. canterbury.ac.nz/ f .voloch 